\title{
Voice and speech outcomes of chemoradiation for advanced head and neck cancer: a systematic review
}

\author{
Irene Jacobi $\cdot$ Lisette van der Molen • \\ Hermelinde Huiskens • Maya A. van Rossum • \\ Frans J. M. Hilgers
}

Received: 12 April 2010 / Accepted: 14 June 2010 / Published online: 30 June 2010

(C) The Author(s) 2010. This article is published with open access at Springerlink.com

\begin{abstract}
Purpose of this review is to systematically assess the effects on voice and speech of advanced head and neck cancer and its treatment by means of chemoradiotherapy (CRT). The databases Medline, Embase and Cochrane were searched (1991-2009) for terms head and neck cancer, chemoradiation, voice and speech rehabilitation. Twenty articles met the inclusion criteria, whereof 14 reported on voice outcomes and 10 on speech. Within the selected 20 studies, 18 different tools were used for speech or voice evaluation. Most studies assessed their data by means of patient questionnaires. Four studies presented outcome measures in more than one dimension. Most studies summarised the outcomes of posttreatment data that were assessed at various points in time after treatment. Except for four studies, pre-treatment measurements were lacking. This and the fact that most studies combined the outcomes of patients with radiated laryngeal cancers with outcome
\end{abstract}

I. Jacobi and L. van der Molen contributed equally to this article.

I. Jacobi $\cdot$ L. van der Molen · F. J. M. Hilgers ( $\square)$

The Netherlands Cancer Institute, Plesmanlaan 121,

1066 CX Amsterdam, The Netherlands

e-mail: f.hilgers@nki.nl

H. Huiskens · F. J. M. Hilgers

Institute of Phonetic Sciences,

University of Amsterdam, Amsterdam, The Netherlands

M. A. van Rossum

Leiden University Medical Center,

Leiden, The Netherlands

F. J. M. Hilgers

Academic Medical Center,

University of Amsterdam, Amsterdam, The Netherlands data of non-laryngeal cancer patients impedes an interpretation in terms of the effects of radiation versus the effects of the disease itself on voice or speech. Overall, the studies indicated that voice and speech degenerated during CRT, improved again 1-2 months after treatment and exceeded pre-treatment levels after 1 year or longer. However, voice and speech measures do not show normal values before or after treatment. Given the large-ranged posttreatment data, missing baseline assessment and the lacking separation of tumour/radiation sites, there is an urgent need for structured standardised multi-dimensional speech and voice assessment protocols in patients with advanced head and neck cancer treated with CRT.

Keywords Head and neck cancer - Organ preservation . Systematic review $\cdot$ Voice $\cdot$ Speech $\cdot$ Rehabilitation

\section{Introduction}

In organ preservation protocols, concomitant chemoradiotherapy (CRT) has become a favored treatment in medically fit patients with advanced head and neck cancer [1]. However, organ preservation unfortunately not always equals function preservation and head and neck cancers and their treatment by chemoradiation induce quite some functional changes, e.g. to the quality of voice and speech. Mapping and reporting these functional changes obviously are an important aspect of the evaluation of organ preservation protocols. Commonly used outcome measures to assess organ functioning before, during and after treatment are oral intake or consistency of diet, swallowing measures and quality of life questionnaires [2]. The functioning of the larynx and the articulators in terms of voice and speech are less commonly reported outcome measures. Nonetheless, 
speech and voice are the most important daily communication tools and part of a person's identity and personality [3] and contribute to the patients' well being and overall quality of life $[4,5]$.

Here, 'voice' is defined as the sound originating from the vibrations of the vocal folds. The quality of voice is thus dependent on the myoelastic characteristics of the vocal folds, and is affected only slightly by the resonances and characteristics of other parts of the vocal tract. Speech on the other hand, as a carrier of messages, is based on the volitional coordinated movements of the articulators and can be affected severely by changes in muscle or tissue properties of e.g. the tongue or the soft palate.

Given the various tumour sites of head and neck cancer, non-laryngeal (i.e. oral cavity, oropharynx, hypopharynx and nasopharynx) and laryngeal cancers, it is expected that the effects of the tumour and its treatment on voice and speech outcomes differ. In non-laryngeal tumours, the tumour itself will not affect voice quality, yet, depending on its location, it can impede speech. In patients with laryngeal tumours, the tumour can have a negative effect on voicing, whereas its treatment can affect both voice and speech. In addition, radiation to lymph nodes can affect voice quality. However, outcomes are often summarised across tumour sites and many studies are not consistent in separating speech and voice problems and terminology.

Next to CRT, other interventions can affect the patients' voice and speech, e.g. early studies show the efficacy of rehabilitative or preventative voice therapy $[6,7]$. Most recently its efficacy has been reported for cases of early cancer [8]. Studies on efficacy of voice/speech therapy is rare, although, especially in cases of advanced head and neck cancer [9].
To analyse the effects of the tumour versus the side effects of radiation, pretreatment data are needed for a proper analysis of the various factors involved. When defining the outcome, the way the data were assessed will play a major role. Which instruments are used to assess voice and speech? In cases of different assessment tools-do the studies show comparable outcomes? Do the measurements cover different dimensions of assessment, such as perception, acoustics, stroboscopy, subjective ratings, or aerodynamics as suggested e.g. by Dejonckere et al. [10]. In addition, what are the effects of speech, voice or swallowing therapy on voice and speech and finally, are there indications of their efficacy in cases of advanced head and neck cancer treated by CRT?

To evaluate the effects of advanced head and neck cancer and its treatment by means of CRT on voice and speech a systematic literature search was carried out. First, an overview of the studies that evaluated effects of advanced head and neck cancer and/or the effects of CRT on voice and speech will be given. Next, the additional questions described above will be answered.

\section{Materials and methods}

A systematic search of the literature was carried out using the electronic databases Medline, Embase and Cochrane. Keywords and Mesh terms (or possible synonyms) included: 'head and neck cancer', 'chemoradiotherapy (CRT)' and terms referring to voice and speech problems and their rehabilitation or assessment (see Fig. 1). Included were studies published from 1991 till November 2009. The search was then limited to studies on human adults (18+ years) and published in English, French or German.
Fig. 1 Overview literature search

\begin{tabular}{|l|l|l|l|}
\hline head and neck cancer & AND & chemotherapy and radiotherapy or chemoradiation or organ \\
preservation or synonyms
\end{tabular}


Three reviewers, IJ, LM and HH, screened the abstracts of all hits, and the full text was retrieved of those that were considered relevant. All articles' references were checked for additional potentially relevant studies. Considered relevant were studies that address speech and voice outcomes after concomitant CRT, and that had not included patients prior to 1990. Studies that only focused on radiotherapy alone and/or surgery were excluded. The study population should consist of at least ten patients with squamous cell carcinoma and T2-4 tumours of the oral cavity, oropharynx, hypopharynx, larynx and/or nasopharynx. All selected studies had to be research based and published in a peerreviewed journal and had to report on voice-, or speechrelated functional problems. The criteria were not met in cases the reported functional outcomes related to solely survival, nutrition, or toxic effects, see Table 1 .

\section{General results}

The search strategy resulted in 231 hits and 17 of the 37 selected articles failed to match the in- and exclusion criteria. Finally, twenty studies were included, see also Fig. 1 [11-30]. In the following, first, the 20 articles will be discussed according to their study design and assessment tools, before they are split according to their voice and speech outcome.

Patient characteristics and study design

Table 2 provides a detailed description of the 20 studied papers. Age, sex, treatment and the site of lesion were reported in all studies. Explicit patient inclusion criteria in view of diagnosis, patient history and physical state were missing or only available implicitly, in 9 of the 20 studies $[13,14,17,19,20,22,24,25,29]$. Four of the 20 articles reported on overall tumour stage only, so without specific tumour classification [19, 20, 22, 24].

When considering the replication potential of the studies with respect to the included patient groups, in most studies, the information was insufficient. Individual data were grouped over various patient attributes and were not separated e.g. with respect to site of tumour/radiation, even in small$\mathrm{N}$ studies. Patients with laryngeal primary cancer were included in 16 studies, hypopharyngeal carcinoma in 9, oropharyngeal in 5, patients with cancer of the oral cavity in 4, and nasopharyngeal cancer patients in 1 study.

Of the 20 studies, 11 mentioned explicitly the period (range of years) of patient accrual [11-15, 17, 21, 23, 2628]. For nine articles, there was no or insufficient information on the range of the patient accrual phase $[16,18-20$, $22,24,25,29,30]$. Fifteen of the 20 articles were prospective studies [11, 12, 14-16, 18, 20, 22, 24-30], 5 were retrospective $[13,17,19,21,23]$. In 8 of the 15 prospective studies, data were gathered at structured points in time after treatment [11, 12, 15, 20, 24, 25, 27, 28].

Four of the prospective studies included baseline and posttreatment measurements, with all data being collected at structured points in time up to 1 year after treatment [12, $20,24,27]$. The rest of the 16 studies reported on posttreatment outcome only. Four of these 16 measured at one specific posttreatment point in time $[11,15,25,28]$ and in 12 studies, data of (large) posttreatment ranges were summarised in the reported outcome (see Fig. 2 for a comparison of the points in time across studies). Five studies reported on a homogenous treatment group of patients $[11,15,23$, $28,30]$, whereas 15 compared different treatment and patient groups.

Instruments

To analyse or compare the different voice and speech outcomes described in the literature, it is important to take into consideration the type of instruments that were used. Within the 20 selected studies, 18 different tools were used for measuring voice and speech after CRT. Table 3 gives an overview of these 18 tools and what kinds of questions were used to assess voice and/or speech. Fourteen studies reported on voice outcomes and 10 on speech. Within these 20 studies, 10 patient-based instruments were used, 3 clinician-based instruments and 3 newly created instruments. Objective measures were used in 10 studies. There were large differences in elaborateness and accuracy of the instruments to assess voice or speech problems.
Table 1 In- and exclusion criteria for relevance of the article

\begin{tabular}{ll}
\hline Inclusion & Exclusion \\
\hline $\begin{array}{l}\text { Oral cavity, oropharynx, hypopharynx, } \\
\text { larynx and/or nasopharynx carcinoma }\end{array}$ & Inclusion of patients $<1,990$ \\
T2-4 squamous cell carcinoma & Population $\leq 10$ \\
Treatment with chemoradiotherapy & Treatment with surgery or radiotherapy alone \\
Outcome measurements of speech and/or Voice & $\begin{array}{c}\text { Outcomes solely about survival, } \\
\text { nutrition or toxic effects }\end{array}$ \\
\hline
\end{tabular}




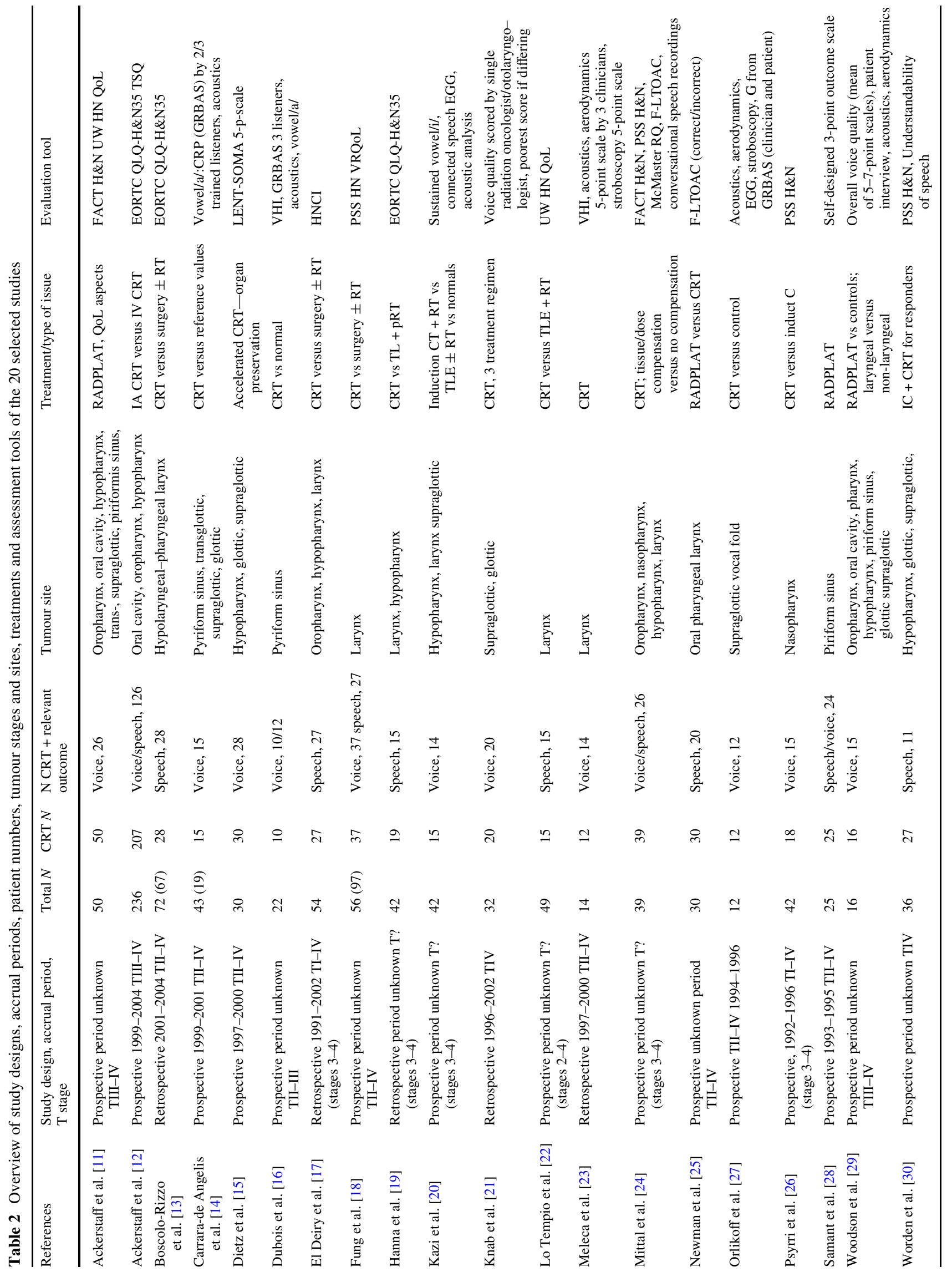




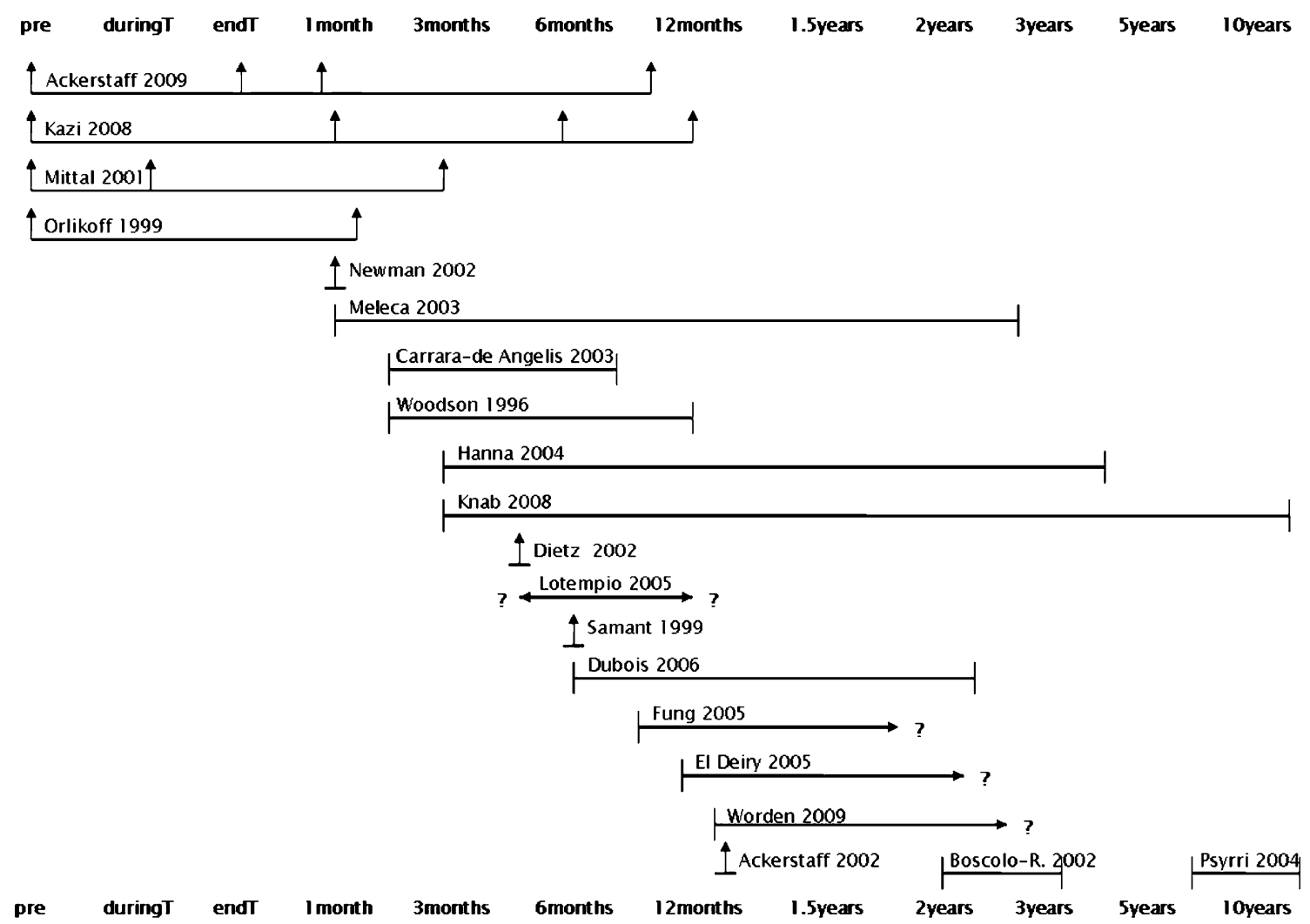

Fig. 2 Pre- and posttreatment data assessment per study; vertical arrows indicate structured points in time of data assessment, horizontal arrows indicate unclear end or beginning of the data assessment range

Integrity of the reported results

All 20 studies report their outcomes by means of statistics. However, especially in cases of self-created instruments, reliability and validity is lacking, and in clinician-based instruments, inter- and intrarater reliability often is not reported.

Only 13 studies had sufficient information on patient- or data-loss [11-13, 16, 17, 19, 21-23, 25, 27-29]. Owing to the lack of report on patient- and data-loss, in the prospective studies, the patient characteristics of the remaining group on which the outcome is based often remains unclear in terms of tumour locations or stages. In three studies, the number of listeners [14] or patient subgroup numbers were reported inconsistently throughout the text $[15,29]$.

Of the selected 20 articles, 3 studies mention voice-, speech- or swallowing therapy in their own patient group [14], or referred to it as something that should be considered in future studies $[18,21]$. For the other studies, it remains unclear whether voice or speech therapy, or swallowing exercises were part of the treatment protocol or not.

Several studies mentioned that (unknown numbers of) patients underwent tracheotomy or gastrostomy prior or during treatment. However, none of the authors mentioned that this also could affect voice and speech outcomes and measurements by e.g. inducing a change in the patient's perception or the efficacy of voice-, speech- or swallowing exercises. Next to alternative nutrition, decreased hydration is known to affect voice quality and effort $[31,32]$, but was mentioned in none of the relevant studies.

\section{Voice and speech outcome}

Only two studies made a distinction between the effects of tumour and of treatment on voice and/or speech outcome [20, 23]. Kazi et al. [20] mentioned that laryngeal tumours distort voice quality because they obstruct the airflow through the glottis, they impair normal cord movement, and they are accompanied by significant oedema. The treatment effects could be radiation-induced fibrosis or vocal fold atrophy and swelling of laryngeal and pharyngeal tissues. Meleca et al. reported that vocal fold neuromuscular weakness and paresis could be a result of the invasive effects of the tumour, whereas fibrosis, mucositis of the laryngeal soft tissues and vocal fold atrophic changes are caused by the treatment [23].

As mentioned before, 16 of the 20 studies had not collected voice or speech data prior to treatment, but reported on posttreatment outcome alone (see Fig. 2). In 12 of these stud- 
Table 3 Overview of the 18 measurement instruments on voice or speech used in the 20 studies, with references in brackets, followed by examples of the questions used and the number of studies that used the tool

\begin{tabular}{|c|c|c|c|}
\hline Type of instrument & Instrument & Questions on voice or speech & Number \\
\hline \multirow[t]{12}{*}{$\begin{array}{l}\text { Patient-based: created } \\
\text { trial-specific } \\
\text { patient-based }\end{array}$} & EORTC QLQ-H\&N35 [37] & $\begin{array}{l}\text { "Have you been hoarse?", } \\
\text { "Have you had trouble talking to other people?", } \\
\text { "Have you had trouble talking on the telephone?" } \\
\text { (all 4-point scales) }\end{array}$ & 3 \\
\hline & VHI [38] & 13 items,on voice (all 5-point scales) & 2 \\
\hline & FACT H\&N [39] & $\begin{array}{l}\text { "My voice has its usual quality and strength" } \\
\text { "I am able to communicate with others" } \\
\text { (both 4-point scales) }\end{array}$ & 2 \\
\hline & HNCI [40] & $\begin{array}{l}10 \text { items on items on peech/communication } \\
\text { and voice (all 5-point scores) }\end{array}$ & 1 \\
\hline & V-RQoL [41] & $\begin{array}{l}10 \text { questions with } 5 \text {-point scores on quality } \\
\text { of voice (plus } 1 \text { overall score) }\end{array}$ & 1 \\
\hline & UW H\&N QoL [42] & One 4-point scale item on speech & 1 \\
\hline & HNRQ [42] & $\begin{array}{l}\text { "Have you had a hoarse voice in the past week?", } \\
\text { plus 6-point scale for "How troublesome was this for you?" }\end{array}$ & 1 \\
\hline & LENT-SOMA [43] & "Is your voice hoarse?", 5-point scale & 1 \\
\hline & TSQ [44] & $\begin{array}{l}\text { 4-point scales on intelligibility, loudness, pitch, fluency, } \\
\text { intelligibility on the telephone (summated Likert's scale) }\end{array}$ & 1 \\
\hline & PSS H\&N [45] & One item on understandability of speech (5-point scale) & 4 \\
\hline & GRBAS [46] & $\begin{array}{l}\text { Grade, roughness, breathiness, asthenia } \\
\text { and strain of voice, all 4-point scales }\end{array}$ & 3 \\
\hline & F-LTOAC [47] & Correctness of articulation & 2 \\
\hline \multirow[t]{3}{*}{ Objective } & Acoustics/EGG & NA & 5 \\
\hline & Aerodynamics & NA & 3 \\
\hline & Videostroboscopy & NA & 2 \\
\hline \multirow{3}{*}{$\begin{array}{l}\text { Created study-specific } \\
\text { clinician-based: }\end{array}$} & Voice quality rating [28] & Three grades & 1 \\
\hline & Voice quality rating [21] & One 4-point scale & 1 \\
\hline & Voice quality rating [29] & Mean of 5 voice parameters, all 7-point scales & 1 \\
\hline
\end{tabular}

NA not applicable

EORTC QLQ-H\&N35 European Organisation for Research and Treatment of Cancer Quality of Life Questionnaire-Head \& Neck 35, VHI Voice Handicap Index, FACT H\&N Functional Assessment of Cancer Therapy-Head and Neck, HNCI Head and Neck Cancer Inventory, V-RQoL voice-related quality of life, $U W H \& N$ QoL University of Washington Head and Neck Quality of Life, HNRQ McMaster Head and Neck Radiotherapy Questionnaire, $L E N T$-SOMA late effect of normal tissue-subjective objective management analytic scoring system, TSQ trial-specific questionnaire, PSS $H \& N$ performance status scale head and neck, GRBAS grade, roughness, breathiness, asthenia, strain scale, F-LTOAC Fisher Logeman Test of Articulation Competence

ies, the outcome was based on the data accrual between 1 month and up to 12 years posttreatment. Overall, the results were reported regardless of (large) differences in the dates of the patients' posttreatment data accrual, except for one study by Fung et al. [18]. Here, the data were included from a minimum of 8 months posttreatment on, with a median of 36.9 months. A significant correlation was found for the time that had passed since treatment and the VRQoL scores.

Given the missing baseline data and the role of time passing since treatment, it is difficult to interpret the outcome of studies that summarise larger posttreatment ranges. Therefore, the following paragraphs will only focus on the outcomes of the studies that included baseline data and assessed their patients' voice and speech functioning at structured points in time after treatment.
Pretreatment voice and speech status (effects of cancer)

Four studies included measurements before treatment [12, 20, 24, 27]. In the study of Mittal et al. [24] that included laryngeal- and non-laryngeal tumours, the focus was on differences between two treatment groups, one with and one without tissue/dose compensation, and on the significant changes between pre- and 3 months posttreatment outcome. Pretreatment values of Performance Status Scale Head \& Neck (PSS H\&N) scores on speech were only mentioned for 2 of 26 patients whose scores were above 50 pretreatment and below 50 at 3 months posttreatment. In terms of voice, eight patients showed no or mild problems in pretreatment that changed to moderate or severe problems at 3 months posttreatment (McMaster Head and Neck Radio- 
therapy Questionnaire, HNRQ). Voice and speech scores of other patients prior to treatment were not reported. In Kazi et al. [20], electroglottography (EGG) showed that the patients pretreatment jitter values were significantly different from normal values. In the CRT patient group $(N=14)$, also, the parameter "words per minute" prior to treatment was significantly lower than in normal subjects $(N=21)$. The CRT patient group included patients with hypopharyngeal, laryngeal and supraglottic laryngeal cancers. In the study of Orlikoff et al. [27], before treatment, all except 2 of 12 patients showed abnormal EGG wave patterns. Most salient were increased contact irregularity, increased contact interval and disruption of vocal fold separation [27]. Mentionable in view of the functioning and status of the voice and speech organs, furthermore, is that eight patients could not be analysed by stroboscopy because they did not tolerate the endoscopic examination. The degree of dysphonia was judged both by the patient and the speech pathologist based on a modified Grade, Roughness, Breathiness, Asthenia, Strain scale (GRBAS). Prior to treatment, seven patients were diagnosed with moderate, and five with severe dysphonia.

In Ackerstaff et al. [12], the European Organisation for Research and Treatment of Cancer Quality of Life Questionnaire-Head and Neck (EORTC H\&N35) outcome of CRT patients (intraarterial (IA) CRT $N=60$; intravenous (IV) CRT $N=66$ ) with cancer of the oral cavity, oropharynx or hypopharynx reveals revealed scores of $18.4 / 18.3$ for speech on a symptom scale of $0-100$. Presumably, 'speech' here included the two questions on talking, and the questions on coughing and hoarseness, thus 'voice'-related items. While the focus of the authors was on posttreatment changes, baseline data on voice that were assessed with a task-specific questionnaire were not reported.

The baseline status of the patients $(N=39)$ with cancer of the larynx, the oro-, naso-, or hypopharynx in Mittal et al. [24] were difficult to interpret, from the EGG data [20, 27] and the patient-based questionnaires [12] it can be concluded that prior to treatment, voice and speech were already impaired compared to normal control subjects.

\section{Short-term posttreatment outcomes}

One study, Ackerstaff et al. [12] reported on early outcomes, assessed at the last day of the 7-week treatment period. The mean of the symptom scale of speech that was $18.4 / 18.3$ on a symptom scale of $0-100$ prior to treatment, increased considerably to 45.3 for the IA group $(N=88)$ and $33.7(N=95)$ for the IV group. Voice quality assessed by means of a task-specific questionnaire on intelligibility, loudness, pitch and fluency deteriorated as well.

Four studies reported on outcomes, approximately 1 month posttreatment $([12,20,25,27]$, with a range of
1-2 months for Orlikoff et al. [27]). Kazi et al. [20] focused on 12 months posttreatment results, but their tables of EGG results on jitter and the number of words per minute show insignificant changes 1 month after treatment compared to pretreatment values. The pretreatment values had been significantly different from those of normal individuals. In Orlikoff et al. [27], when compared with normal controls, phonation remained abnormal 1-2 months posttreatment when compared with the phonation of normal controls. However, 9 of 12 patients showed "substantial improvement" in voice quality. When compared with pretreatment EGG values, vocal stability in terms of jitter improved significantly, as do the pitch range and airflow measures, the latter indicating improved glottal function and air management. The third study with 1-month posttreatment data by Newman et al. [25] scored articulation by means of the sentence version of the Fisher-Logeman test of Articulation Competence (F-LTOAC) (correct/incorrect) in IA versus IV CRT patients. The outcome reveals overall scores for percentage of consonants articulated correctly of $79.9 \%$ (IA CRT) and 96.6\% (IV CRT), with the worst scores for alveolar fricatives. The authors mentioned that the differences were adjusted for baseline differences between groups. Thus, pretreatment data on articulation seems to have been available, but was not reported. The poorer results of the IA group were explained by the fact that the group included $\mathrm{T}$ stages that were more advanced, contained more oral cancer, and edentulous patients than the CRT group. In the last study [12], five weeks after the end of treatment, the mean speech score had improved and exceeded pretreatment levels.

In summary, voice and speech deteriorated during treatment [12], and, based on the three studies that compared their posttreatment outcome with pretreatment measures [12, 20,27], voice and speech seem to improve 1-2 months after treatment, exceeding pretreatment levels.

\section{1-year posttreatment outcomes}

1 year after initiating treatment, in the patient groups of Ackerstaff et al. [12], the mean speech score had further improved. Eighty percent of the patients reported a reasonable good voice, but, due to hoarseness and a dry mouth, for only $33 \%$ their voice and talking was as it used to be. In an earlier study of Ackerstaff et al. [11, $N=26], 1$ year after initiating treatment of the surviving patients who had no surgery, $61 \%$ reported a normal voice, $27 \%$ a somewhat normal voice and $12 \%$ a voice far from what it used to be. No pretreatment data were available in this study.

In Kazi et al. [20], in the first year after treatment, EGG vowel parameters and connected speech measures showed progressive improvement towards normal levels. At 12 months after treatment, the rate of speech in the CRT 
group $(N=14)$ recovered and was equivalent to those of normal control subjects. However, although changing (significantly) towards normal, some voice quality parameters such as the 'larynx frequency distribution' ('DFx1'; an EGG measure of the fundamental frequency in $\mathrm{Hz}$ ) remained abnormal.

Voice and speech thus seem to further improve in the second half year after treatment, although normal values of voice quality were not reached.

\section{Long-term posttreatment outcomes}

Long-term outcomes assessed later than 1 year posttreatment are included in nine studies [13, 16-19, 21, 23, 26, $30]$. In four of these [16, 19, 21, 23], however, the outcomes of early and late assessment were averaged, while the data had been assessed from as early on as 1-8 month posttreatment (see Fig. 2).

Three studies reported on measurements from approximately 12 months posttreatment [13, 17, 26, 30]. While the end of the data assessment was unclear in two of these studies [17, 30], the other two studies covered clearly delimited posttreatment assessment periods. Because there were no studies on long-term effects that included pretreatment data, there were no reliable outcomes of long-term treatment effects. However, the data assessment of these studies started at least 2 years after treatment, so that at least early treatment effects were excluded and, with precaution, a closer look on the two studies with delimited assessment periods might give some insight into late treatment effects: In Boscolo-Rizzo et al. [13], the results of a EORTC QLQ-H\&N35 in a group of 28 laryngeal or hypopharyngeal cancer patients were gathered 2-3 years posttreatment. The outcome on the questionnaire revealed a median speech score of 0.0 with a range of $0-78$, thus indicating that for the large majority, speech was not impaired, with probably a few outliers with serious speech problems. Since the EORTC QLQ-H\&N35 includes only one item on voice, presumably, overall, voice quality was not remarkably abnormal either for the majority of patients. In the second study, by Psyrri et al. [26], quality of life data (PSS H\&N) was available 7-11 years posttreatment. The authors reported persistent hoarseness in 2 of 18 patients, whereas 3 patients report their voice quality had improved. Since the PSS H\&N has one question on speech and no item on voice, the origin of the voice outcome remains unclear.

In the long-term measurements, the treatment effects seem to have decreased. Voice and speech quality seem to be better than in the short-term data. However, there still seem to be cases of severe dysfunction of voice and speech. On the account of the missing baseline information, these long-term results have to be interpreted with caution.
More dimensional assessment

Four studies analysed their data in more than one dimension. All focused on voice outcomes. In Carrara-de Angelis et al. [14], the acoustic measurements correlated significantly with the perceptual degree of dysphonia as judged by clinicians. In contrast, Woodson et al. [29] concluded that acoustic measures did not necessarily reflect how patients perceived communication in daily life. Their patients' judgments of vocal functioning, on the other hand, did correlate with the clinicians' perception. This again was not in line with the findings in Meleca et al. [23], who compared directly the outcome of three dimensions. While expert listeners were in agreement with the results from acoustic and aerodynamic measurements, patient judgments deviated. With regards to more dimensional assessment, Orlikoff et al. [27] in their discussion emphasised that there is a discrepancy between acoustic, clinician-based and patientbased data. Whereas subjective long-term data seem to indicate continued voice improvement, clinician-based assessments describe a continued worsening. There were no studies that addressed more dimensional assessment of speech functioning.

\section{Discussion and conclusion}

The purpose of this review was to find out what impact the tumour and chemoradiation has on voice and/or speech in patients with advanced head and neck cancer.

The literature search that was carried out for this review revealed only few studies that measured voice and/or speech. Of these, a total of 20 studies met the inclusion criteria. Although publications from 1990 till November 2009 were included, thus covering a period of almost 20 years, the studies that met the criteria were almost all published within the last 10 years. This indicates that voice and speech outcomes were of rather secondary interest in earlier organ preservation protocols suggesting that after organ preservation intact function preservation was taken for granted. It seems that only in the last decade clinicians have come to realise that organ and function preservation are not necessarily synonymous.

Of the retrieved hits, most studies focused on the overall survival or toxic effects, usually comparing organ preservation with surgical treatment. There were hardly studies that focused on findings or variations within a homogeneous CRT group of patients, and often, the patient groups included patients that also underwent surgery.

Of the 20 included studies, 14 reported on voice and 10 on speech, an important distinction that too seldom is made. CRT patients scored worse on the voice and speech scores as compared to normal laryngeal speakers, but better as 
compared to patients who received surgical treatment. In general, the preference of CRT over surgery combined with radiotherapy thus seems to be justified, although there were still severe negative effects. Overall, the studies indicated that voice and speech degenerated during CRT treatment, and improved again 1-2 months after treatment, exceeding pre-treatment levels after 1 year or longer. However, voice and speech measures did not show normal values, neither before, nor after treatment.

More data and studies that are more precise and that include pretreatment measurements are needed to evaluate posttreatment voice and speech quality in the long run. Given unexplained dropouts, unclear follow ups, missing reliability and validity of the tools, no intra- and interrater checks, missing baseline measures, various and small number of patients, unknown accrual times, and the lack of uniformity between the studies, the findings were not very reliable. Next to this, the replication potential was small.

Only one of the studies discussed in this review made explicitly a difference between tumours originating from the oral cavity and pharyngeal area and tumours originating from the larynx [29]. The merged results of laryngeal and non-laryngeal cancer patients (and lacking information on radiation to the lymph nodes) make any interpretation of alternations of voice and speech due to cancer and the treatment by various CRT difficult. Only two studies made a distinction between the effects of tumour and treatment [20, 23].

In laryngeal cancers, one would expect the tumour to impede vocal fold movement, resulting in deteriorated voice quality. In non-laryngeal cancers, one would expect the tumour to have a negative effect on articulation, and therewith speech. With the treatment-induced shrinkage of the tumour, voice and speech, respectively, should improve, and lasting negative effects would be attributed to inherent anatomical changes (e.g. scars), radiation oedema and/or fibrosis. One goal of this review was to systematically assess and disentangle these effects of the tumour and its treatment by CCRT on voice and speech. However, due to the inconsistent information in the studies on e.g. tumour location, this aim could not be met.

Several factors were left unmentioned, such as the effects of speech-, voice- or swallowing therapy, tracheotomy, gastrostomy or radiotherapy to the salivary glands or lymph nodes. Furthermore, not only there was a large range in follow-up periods, also, in several studies, the follow-up time metering started with the initiation of treatment, whereas in other studies, metering started with the end of treatment, making a comparison more cumbersome.

There were 18 different measurement instruments and most of these tools are quality of life measures, assessing voice and speech outcomes rather superficially. Especially the standardised, validated questionnaires are "poorly equipped' with voice and speech items, underlining the importance of (also) using study/topic specific questionnaires [33]. In addition, in general, terminology and assessment of voice and speech problems are often incompletely separated. This review underlines that the 18 tools used not only showed a wide variation (acoustic/EGG measurements, and patient-based, and/or clinician-based questionnaires), but also that several of these tools were not standardised, and often, interrater reliability and/or validity were not reported.

Almost all studies based their outcome on one-dimensionally assessed data; the most favored method of assessment was the patient questionnaire. Given the often limited number of patients, this is probably the 'easiest' method of voice and speech quality assessment, as no clinician or equipment needs to be involved. Although these questionnaires provide a relevant view on the patient's perceived quality of life, they do not necessarily reflect the patient's actual physical status, and organ functioning, or the clinician's perception. There is a lack of studies that compare the outcome of voice- and in particular speech in different assessment dimensions to verify the extent or feasibility for each subjective or objective dimension of measurement, and its clinical indication.

This systematic review showed that the need for more dimensional assessment of organ functioning was hardly ever mentioned, although it is obvious that multi-dimensional assessment is mandatory [23, 27, 34].

Proposed protocols are available for the analysis of voice, e.g. by Verdonck-de Leeuw et al. [35], Meleca et al. [23], or Dejonckere et al. [10]. All agree in suggesting a multi-dimensional subjective and objective analysis. Suggestions for speech assessment protocols for cancer patients are not yet available, but certainly needed, because it is obvious that tumour- or treatment-related articulatory disorders strongly affect the intelligibility of speech. Usually, clinician- and/or patient-based tools are used to assess intelligibility and articulatory abilities of the patient. However, human perception always carries a subjective imprint, and a clinician-based analysis with various listeners is rather time expensive and impractical in a clinical setting. Therefore, comparable to the proposed multi-dimensional protocols for voice analysis, a similar tool for the analysis of speech should include at least a patient questionnaire on speech and intelligibility, together with acoustic analyses and e.g. the F-LTOAC. In future, if they succeed in copying the behaviour of either a normal representative listener or that of an experienced clinician, automatic speech intelligibility analyses, which are less costly in time, might help in this respect [36].

The studies mentioned in this systematic review provide only a superficial picture of the effects of cancer and CRT on voice and speech in patients with advanced head and 
neck cancer. Considering the changes of voice and speech quality posttreatment, more and more precise, preferably prospective studies are needed, including both baseline measurements and a standardised assessment protocol that covers all relevant functional aspects of voice and speech.

Open Access This article is distributed under the terms of the Creative Commons Attribution Noncommercial License which permits any noncommercial use, distribution, and reproduction in any medium, provided the original author(s) and source are credited.

\section{References}

1. Cognetti DM, Weber RS, Lai SY (2008) Head and neck cancer: an evolving treatment paradigm. Cancer 113:1911-1932

2. van der Molen L, van Rossum MA, Burkhead LM, Smeele LE, Hilgers FJ (2009) Functional outcomes and rehabilitation strategies in patients treated with chemoradiotherapy for advanced head and neck cancer: a systematic review. Eur Arch Otorhinolaryngol 266:901-902

3. Rosen DC, Sataloff RT (1997) Voice disorders; psychological aspects; patients; rehabilitation; psychology. Singular Publication Group, San Diego

4. Peeters AJ, van Gogh CD, Goor KM, Verdonck-de Leeuw IM, Langendijk JA, Mahieu HF (2004) Health status and voice outcome after treatment for T1a glottic carcinoma. Eur Arch Otorhinolaryngol 261:534-540

5. Fung K, Yoo J, Leeper HA, Bogue B, Hawkins S, Hammond JA, Gilchrist JA, Venkatesan VM (2001) Effects of head and neck radiation therapy on vocal function. J Otolaryngol 30:133-139

6. Fex S, Henriksson B (1969) Phoniatric treatment combined with radiotherapy of laryngeal cancer for the avoidance of radiation damage. Acta Otolaryngol Suppl 263:128-129

7. Lehman JJ, Bless DM, Brandenburg JH (1988) An objective assessment of voice production after radiation therapy for stage I squamous cell carcinoma of the glottis. Otolaryngol Head Neck Surg 98:121-129

8. van Gogh CD, Verdonck-de Leeuw IM, Boon-Kamma BA, Rinkel RN, de Bruin MD, Langendijk JA, Kuik DJ, Mahieu HF (2006) The efficacy of voice therapy in patients after treatment for early glottic carcinoma. Cancer 106:95-105

9. Lazarus CL (2009) Effects of chemoradiotherapy on voice and swallowing. Curr Opin Otolaryngol Head Neck Surg 17:172-178

10. Dejonckere PH, Bradley P, Clemente P, Cornut G, Crevier-Buchman L, Friedrich G, Van De Heyning P, Remacle M, Woisard V (2001) A basic protocol for functional assessment of voice pathology, especially for investigating the efficacy of (phonosurgical) treatments and evaluating new assessment techniques. Guideline elaborated by the Committee on Phoniatrics of the European Laryngological Society (ELS). Eur Arch Otorhinolaryngol 258:77-82

11. Ackerstaff AH, Tan IB, Rasch CR, Balm AJ, Keus RB, Schornagel JH, Hilgers FJ (2002) Quality-of-life assessment after supradose selective intra-arterial cisplatin and concomitant radiation (RADPLAT) for inoperable stage IV head and neck squamous cell carcinoma. Arch Otolaryngol Head Neck Surg 128:11851190

12. Ackerstaff AH, Balm AJ, Rasch CR, de Boer JP, Wiggenraad R, Rietveld DH, Gregor RT, Kroger R, Hilgers FJ (2009) First-year quality of life assessment of an intra-arterial (RADPLAT) versus intravenous chemoradiation phase III trial. Head Neck 31:77-84

13. Boscolo-Rizzo P, Maronato F, Marchiori C, Gava A, Da Mosto MC (2008) Long-term quality of life after total laryngectomy and postoperative radiotherapy versus concurrent chemoradiotherapy for laryngeal preservation. Laryngoscope 118:300-306

14. Carrara-de Angelis E, Feher O, Barros AP, Nishimoto IN, Kowalski LP (2003) Voice and swallowing in patients enrolled in a larynx preservation trial. Arch Otolaryngol Head Neck Surg 129:733-738

15. Dietz A, Nollert J, Eckel H, Volling P, Schroder M, Staar S, Conradt C, Helmke B, Dollner R, Muller RP, Wannenmacher M, Weidauer H, Rudat V (2002) Organ preservation in advanced laryngeal and hypopharyngeal carcinoma by primary radiochemotherapy. Results of a multicenter phase II study. HNO 50:146-154

16. Dubois MD, Crevier-Buchman L, Martin C, Prades JM (2006) Epidermoid carcinoma of piriform sinus after chemo-radiotherapy: acoustic evaluation and voice handicap. Rev Laryngol Otol Rhinol (Bord) 127:299-304

17. El Deiry M, Funk GF, Nalwa S, Karnell LH, Smith RB, Buatti JM, Hoffman HT, Clamon GH, Graham SM, Trask DK, Dornfeld KJ, Yao M (2005) Long-term quality of life for surgical and nonsurgical treatment of head and neck cancer. Arch Otolaryngol Head Neck Surg 131:879-885

18. Fung K, Lyden TH, Lee J, Urba SG, Worden F, Eisbruch A, Tsien C, Bradford CR, Chepeha DB, Hogikyan ND, Prince ME, Teknos TN, Wolf GT (2005) Voice and swallowing outcomes of an organpreservation trial for advanced laryngeal cancer. Int J Radiat Oncol Biol Phys 63:1395-1399

19. Hanna E, Sherman A, Cash D, Adams D, Vural E, Fan CY, Suen JY (2004) Quality of life for patients following total laryngectomy vs chemoradiation for laryngeal preservation. Arch Otolaryngol Head Neck Surg 130:875-879

20. Kazi R, Venkitaraman R, Johnson C, Prasad V, Clarke P, RhysEvans P, Nutting CM, Harrington KJ (2008) Electroglottographic comparison of voice outcomes in patients with advanced laryngopharyngeal cancer treated by chemoradiotherapy or total laryngectomy. Int J Radiat Oncol Biol Phys 70:344-352

21. Knab BR, Salama JK, Solanki A, Stenson KM, Cohen EE, Witt ME, Haraf DJ, Vokes EE (2008) Functional organ preservation with definitive chemoradiotherapy for T4 laryngeal squamous cell carcinoma. Ann Oncol 19:1650-1654

22. LoTempio MM, Wang KH, Sadeghi A, Delacure MD, Juillard GF, Wang MB (2005) Comparison of quality of life outcomes in laryngeal cancer patients following chemoradiation vs. total laryngectomy. Otolaryngol Head Neck Surg 132:948-953

23. Meleca RJ, Dworkin JP, Kewson DT, Stachler RJ, Hill SL (2003) Functional outcomes following nonsurgical treatment for advanced-stage laryngeal carcinoma. Laryngoscope 113:720-728

24. Mittal BB, Kepka A, Mahadevan A, Kies M, Pelzer H, List MA, Rademaker A, Logemann J (2001) Tissue/dose compensation to reduce toxicity from combined radiation and chemotherapy for advanced head and neck cancers. Int J Cancer 96 Suppl:61-70

25. Newman LA, Robbins KT, Logemann JA, Rademaker AW, Lazarus CL, Hamner A, Tusant S, Huang CF (2002) Swallowing and speech ability after treatment for head and neck cancer with targeted intraarterial versus intravenous chemoradiation. Head Neck 24:68-77

26. Psyrri A, Kwong M, DiStasio S, Lekakis L, Kassar M, Sasaki C, Wilson LD, Haffty BG, Son YH, Ross DA, Weinberger PM, Chung GG, Zelterman D, Burtness BA, Cooper DL (2004) Cisplatin, fluorouracil, and leucovorin induction chemotherapy followed by concurrent cisplatin chemoradiotherapy for organ preservation and cure in patients with advanced head and neck cancer: long-term follow-up. J Clin Oncol 22:3061-3069

27. Orlikoff RF, Kraus DH, Budnick AS, Pfister DG, Zelefsky MJ (1999) Vocal function following successful chemoradiation treatment for advanced laryngeal cancer: preliminary results. Phonoscope 2:67-77 
28. Samant S, Kumar P, Wan J, Hanchett C, Vieira F, Murry T, Wong FS, Robbins KT (1999) Concomitant radiation therapy and targeted cisplatin chemotherapy for the treatment of advanced pyriform sinus carcinoma: disease control and preservation of organ function. Head Neck 21:595-601

29. Woodson GE, Rosen CA, Murry T, Madasu R, Wong F, Hengesteg A, Robbins KT (1996) Assessing vocal function after chemoradiation for advanced laryngeal carcinoma. Arch Otolaryngol Head Neck Surg 122:858-864

30. Worden FP, Moyer J, Lee JS, Taylor JM, Urba SG, Eisbruch A, Teknos TN, Chepeha DB, Prince ME, Hogikyan N, Lassig AA, Emerick K, Mukherji S, Hadjiski L, Tsien CI, Miller TH, Wallace NE, Mason HL, Bradford CR, Wolf GT (2009) Chemoselection as a strategy for organ preservation in patients with T4 laryngeal squamous cell carcinoma with cartilage invasion. Laryngoscope 119:1510-1517

31. Verdolini K, Titze IR, Fennell A (1994) Dependence of phonatory effort on hydration level. J Speech Hear Res 37:1001-1007

32. Verdolini K, Min Y, Titze IR, Lemke J, Brown K, van Mersbergen M, Jiang J, Fisher K (2002) Biological mechanisms underlying voice changes due to dehydration. J Speech Lang Hear Res 45:268-281

33. Op de Coul BM, Hilgers FJ, Balm AJ, Tan IB, van den Hoogen FJ, van Tinteren H (2000) A decade of postlaryngectomy vocal rehabilitation in 318 patients: a single Institution's experience with consistent application of provox indwelling voice prostheses. Arch Otolaryngol Head Neck Surg 126:1320-1328

34. Friedrich G, Dejonckere PH (2005) The voice evaluation protocol of the European Laryngological Society (ELS)—first results of a multicenter study. Laryngorhinootologie 84:744-752

35. Verdonck-de Leeuw IM, Hilgers FJ, Keus RB, Koopmans-van Beinum FJ, Greven AJ, de Jong JM, Vreeburg G, Bartelink H (1999) Multidimensional assessment of voice characteristics after radiotherapy for early glottic cancer. Laryngoscope 109:241-248

36. Middag C, Martens J, Van Nuffelen G, De Bodt M (2009) Automated intelligibility assessment of pahtological speech using phonological features. EURASIP J Adv Signal Process 1-9
37. Bjordal K, Hammerlid E, Ahlner-Elmqvist M, de Graeff A, Boysen M, Evensen JF, Biorklund A, de Leeuw JR, Fayers PM, Jannert M, Westin T, Kaasa S (1999) Quality of life in head and neck cancer patients: validation of the European Organization for Research and Treatment of Cancer Quality of Life QuestionnaireH\&N35. J Clin Oncol 17:1008-1019

38. Jacobson BH, Johnson A, Grywalski C, Silbergleit A, Jacobson G, Benninger M, Newman C (1997) The voice handicap index (VHI): development and validation. Am J Speech Lang Pathol 6:66-70

39. Cella DF, Tulsky DS, Gray G, Sarafian B, Linn E, Bonomi A, Silberman M, Yellen SB, Winicour P, Brannon J (1993) The functional assessment of cancer therapy scale: development and validation of the general measure. J Clin Oncol 11:570-579

40. Funk GF, Karnell LH, Christensen AJ, Moran PJ, Ricks J (2003) Comprehensive head and neck oncology health status assessment. Head Neck 25:561-575

41. Hogikyan ND, Sethuraman G (1999) Validation of an instrument to measure voice-related quality of life (V-RQOL). J Voice 13:557-569

42. Hassan SJ, Weymuller EA Jr (1993) Assessment of quality of life in head and neck cancer patients. Head Neck 15:485-496

43. Denis F, Garaud P, Bardet E, Alfonsi M, Sire C, Germain T, Bergerot P, Rhein B, Tortochaux J, Oudinot P, Calais G (2003) Late toxicity results of the GORTEC 94-01 randomized trial comparing radiotherapy with concomitant radiochemotherapy for advancedstage oropharynx carcinoma: comparison of LENT/SOMA, RTOG/EORTC, and NCI-CTC scoring systems. Int J Radiat Oncol Biol Phys 55:93-98

44. Ackerstaff AH, Lindeboom JA, Balm AJ, Kroon FH, Tan IB, Hilgers FJ (1998) Structured assessment of the consequences of composite resection. Clin Otolaryngol Allied Sci 23:339-344

45. List MA, Ritter-Sterr C, Lansky SB (1990) A performance status scale for head and neck cancer patients. Cancer 66:564-569

46. Hirano M, McCormick KR (1981) Clinical examination of voice. Springer, New York

47. Fisher HB, Logemann JA (1971) The Fisher-Logemann test of articulation competence. Houghton Mifflin Co, Boston 